OPEN ACCESS

Edited by: Luis F. Callado,

Universidad del País Vasco, Spain

Reviewed by:

Lucia Ciranna,

Università degli Studi di Catania, Italy

Luigia Trabace,

University of Foggia, Italy

*Correspondence:

Samir Haj-Dahmane

dahmane@ria.buffalo.edu

Specialty section: This article was submitted to

Neuropharmacology,

a section of the journal

Frontiers in Pharmacology

Received: 11 June 2018 Accepted: 28 September 2018 Published: 16 October 2018

Citation:

Wang R, Hausknecht $K$, Shen $R-Y$ and Haj-Dahmane S (2018)

Potentiation of Glutamatergic Synaptic Transmission Onto Dorsal Raphe Serotonergic Neurons in the Valproic Acid Model of Autism. Front. Pharmacol. 9:1185. doi: 10.3389/fphar.2018.01185

\section{Potentiation of Glutamatergic Synaptic Transmission Onto Dorsal Raphe Serotonergic Neurons in the Valproic Acid Model of Autism}

\author{
Ruixiang Wang ${ }^{1,2}$, Kathryn Hausknecht ${ }^{1}$, Roh-Yu Shen ${ }^{1,3,4}$ and Samir Haj-Dahmane ${ }^{1,3,4 *}$ \\ ${ }^{1}$ Research Institute on Addictions, University at Buffalo, The State University of New York, Buffalo, NY, United States, \\ ${ }^{2}$ Department of Psychology, University at Buffalo, The State University of New York, Buffalo, NY, United States, ${ }^{3}$ Department \\ of Pharmacology and Toxicology, The Jacob School of Medicine and Biomedical Sciences, University at Buffalo, The State \\ University of New York, Buffalo, NY, United States, ${ }^{4}$ Neuroscience Program, University at Buffalo, The State University \\ of New York, Buffalo, NY, United States
}

Autism spectrum disorder (ASD) is characterized by social and communicative impairments and increased repetitive behaviors. These symptoms are often comorbid with increased anxiety. Prenatal exposure to valproic acid (VPA), an anti-seizure and mood stabilizer medication, is a major environmental risk factor of ASD. Given the important role of the serotonergic (5-HT) system in anxiety, we examined the impact of prenatal VPA exposure on the function of dorsal raphe nucleus (DRn) 5-HT neurons. We found that male rats prenatally exposed to VPA exhibited increased anxiety-like behaviors revealed by a decreased time spent on the open arms of the elevated plus maze. Prenatal VPA exposed rats also exhibited a stereotypic behavior as indicated by excessive self-grooming in a novel environment. These behavioral phenotypes were associated with increased electrical activity of putative DRn 5-HT neurons recorded in vitro. Examination of underlying mechanisms revealed that prenatal VPA exposure increased excitation/inhibition ratio in synapses onto these neurons. The effect was mainly mediated by enhanced glutamate but not GABA release. We found reduced paired-pulse ratio (PPR) of evoked excitatory postsynaptic currents (EPSCs) and increased frequency but not amplitude of miniature EPSCs in VPA exposed rats. On the other hand, presynaptic GABA release did not change in VPA exposed rats, as the PPR of evoked inhibitory postsynaptic currents was unaltered. Furthermore, the spiketiming-dependent long-term potentiation at the glutamatergic synapses was occluded, indicating glutamatergic synaptic transmission is maximized. Lastly, VPA exposure did not alter the intrinsic membrane properties of DRn 5-HT neurons. Taken together, these results indicate that prenatal VPA exposure profoundly enhances glutamatergic synaptic transmission in the DRn and increases spontaneous firing in DRn 5-HT neurons, which could lead to increased serotonergic tone and underlie the increased anxiety and stereotypy after prenatal VPA exposure.

Keywords: autism, valproic acid, anxiety, dorsal raphe nucleus, 5-HT, glutamatergic synapse, E/I imbalance, hyperserotonemia 


\section{INTRODUCTION}

Autism spectrum disorder (ASD) is a prevalent and serious neurodevelopmental disorder, which is characterized by social impairments, deficits in communication skills, restricted social interests, and repetitive behaviors (American Psychiatric Association, 2013). Moreover, ASD is often comorbid with other mental health problems, including excessive anxiety and fear (White et al., 2009). Work conducted over the last decades has shown that ASD is a highly heritable disorder and identified approximately 800 mutated genes as potent risk factors (Abrahams and Geschwind, 2008; Abrahams et al., 2013; Tick et al., 2016). These genes control synaptogenesis and the function of several neurotransmitter systems, including the serotonergic (5-HT) system. Indeed, early studies have reported hyperserotonemia, i.e., elevated whole blood serotonin levels, as the first common biomarker found in ASD patients (Schain and Freedman, 1961; Muller et al., 2016). A meta-analysis showed elevated 5-HT blood levels in $28.3 \%$ of autistic individuals (Gabriele et al., 2014). Hyperserotonemia is also associated with increased repetitive and anxiety-like behaviors (Cook et al., 1994; Muller et al., 2016). Moreover, 5-HT-related gene variants have been linked to ASD. For example, mutations of tryptophan hydroxylase-2 and the short allelic variant of 5-HTTLPR, a polymorphic region on the 5-HT transporter (5-HTT) gene SLC6A4, have been identified as genetic risk factors for ASD (Devlin et al., 2005; Huang and Santangelo, 2008; Arieff et al., 2010). The role of the 5-HT system in ASD is also supported by animal studies showing that mutation of the 5-HTT gene (Muller et al., 2016) or deletion of monoamine oxidase type A (MAOA) leads to ASD-like phenotypes (Gobbi et al., 2005). Collectively, these human and animal studies support a critical role of 5-HT neurotransmission in ASD, which may mediate the increased anxiety phenotype.

In addition to the genetic factors, growing evidence indicates that prenatal and early postnatal environmental factors contribute to ASD (Landrigan, 2010; Grabrucker, 2013; Ornoy et al., 2015). Indeed, prenatal and postnatal exposure to various teratogens, such as valproic acid (VPA), has been shown to profoundly increase the risk for ASD. In humans, exposure to VPA during the first trimester of the gestational period enhances the incidence of ASD in the offspring (Christensen et al., 2013). Similarly, animal studies have consistently reported that prenatally (on embryonic days 11-12.5) VPA-exposed rats exhibit neurological and behavioral deficits similar to human ASD. Therefore, prenatal VPA exposure in rats has been used as a valid animal model to elucidate the neuronal mechanisms mediating behavioral deficits in ASD (Schneider and Przewłocki, 2005; Roullet et al., 2013).

Despite the role of 5-HT neurotransmission in the pathophysiology of ASD, it remains unknown whether the function of dorsal raphe nucleus (DRn) 5-HT neurons, a major source of 5-HT in the brain (Dahlström and Fuxe, 1964; Descarries et al., 1982), is altered in an animal model of ASD. In this study, we examined the function of putative DRn 5-HT neurons in the VPA model of ASD. We found that prenatal VPA exposure increased anxiety-like behaviors in male rats. These behavioral effects were associated with activation of DRn 5-HT neurons and potentiation of glutamatergic synaptic transmission onto DRn 5-HT neurons. Together, these results help unravel previously unsuspected effects of prenatal VPA exposure on the function of glutamatergic synapses onto DRn 5-HT neurons.

\section{MATERIALS AND METHODS}

\section{Animals and Prenatal VPA Exposure}

Rats were bred in house. Briefly, Long Evans male and virgin female breeders (Envigo, Indianapolis, IN, United States) were housed in pairs in breeding cages, food and water ad lib. The colony room was maintained with a $12 \mathrm{~h} / 12 \mathrm{~h}$ light/dark cycle (light on at 7:00 $\mathrm{AM}-7: 00 \mathrm{PM}$ ), temperature at $20-26^{\circ} \mathrm{C}$ and humidity at $40-60 \%$. Rat droppings were carefully monitored on a daily basis until copulatory vaginal plugs were unequivocally identified (embryonic day 1). Then pregnant females were singly housed in standard plastic cages. VPA (sodium salt) was administered (400 mg/kg, s.c., dissolved in saline at $100 \mathrm{mg} / \mathrm{ml}$; vehicle: $4 \mathrm{ml} / \mathrm{kg}$ saline) on embryonic day 12.5. The VPA dose and exposure window were similar to those used in other studies (Roullet et al., 2013; Nicolini and Fahnestock, 2018). The pups were weaned on postnatal day (PD) 21, and then same-sex rats were housed in pairs in standard plastic cages until the completion of the experiments. Only male rats were used in the present study. Electrophysiological recordings were conducted at the age of 6-8 weeks, and behavioral tests were performed at the age of 8 weeks. All the procedures were carried out with approved protocols from the University at Buffalo Institutional Animal care and Use Committee and were in accordance with the National Institutes of Health guidelines for the Care and Use of Laboratory Animals.

\section{Self-Grooming Test}

The self-grooming test was performed in an open field plastic box $(55 \mathrm{~cm} \times 38 \mathrm{~cm} \times 42 \mathrm{~cm})$, during the dark phase of the light/dark cycle. Activity of rats was recorded by an infrared digital camera mounted above the box and connected to a computer. The rat was allowed to habituate to the dimly lit testing room for $15 \mathrm{~min}$, and then was placed in the center of the open field. Recording lasted for $5 \mathrm{~min}$, and duration of self-grooming was measured by manually coding the video clip by individuals blind to the experimental groups. Longer duration of self-grooming in a novel environment indicates greater anxiety in the rat (Gispen and Isaacson, 1981; Kalueff and Tuohimaa, 2005).

\section{Elevated Plus Maze (EPM) Test}

The EPM test was conducted during the dark phase. The wood maze was elevated $50.8 \mathrm{~cm}$ from the floor, with two open arms and two closed arms $(50.8 \mathrm{~cm} \times 10.15 \mathrm{~cm}$ for each of the $4 \mathrm{arms})$. The closed arms were fenced by $50.8 \mathrm{~cm}$-tall transparent Plexiglas walls. Activity of rats was recorded by an infrared digital camera mounted above the maze and connected to a computer. Animals were allowed to habituate to the dimly lit testing room for 15 min prior to the test, and then placed on the center of the maze with the head facing an open arm. Each 
recording lasted for $5 \mathrm{~min}$, and the video clip was manually coded later by individuals blind to the experimental groups. The rat's entries into and durations of stay/travel on the open and closed arms were analyzed. The numbers of entries indicate the animal's locomotion level, more entries suggesting higher locomotor activity. The time spent on the open arms indicates the magnitude of anxiety. A shorter stay on the open arms is indicative of augmented anxiety (Hogg, 1996; Rodgers and Dalvi, 1997).

\section{Brain Slice Preparation}

Brain slices containing the DRn were prepared using previously described procedures (Haj-Dahmane, 2001). In brief, rats were anesthetized with $0.5 \%$ isoflurane and killed by decapitation. A block of the brainstem area containing the DRn was isolated and coronal slices $(300-350 \mu \mathrm{m})$ were cut using a vibratome (Lancer series 1000, Leica Biosystem, St Louis, MO, United States) in ice-cold modified artificial cerebrospinal fluid (ACSF) of the following composition (in $\mathrm{mM}$ ): 110 choline-Cl; $2.5 \mathrm{KCl} ; 0.5 \mathrm{CaCl}_{2} ; 7 \mathrm{MgSO}_{4} ; 1.25 \mathrm{NaH}_{2} \mathrm{PO}_{4} ; 26.2 \mathrm{NaHCO}_{3}$; 11.6 sodium L-ascorbate; 3.1 sodium pyruvate; and 25 glucose, equilibrated with $95 \% \mathrm{O}_{2} / 5 \% \mathrm{CO}_{2}$. Slices were incubated for $45 \mathrm{~min}$ at $35^{\circ} \mathrm{C}$ and then at room temperature for at least $1 \mathrm{~h}$ in a holding chamber containing standard ACSF (in mM): $119 \mathrm{NaCl} ; 2.5 \mathrm{KCl} ; 2.5 \mathrm{CaCl}_{2} ; 1.3 \mathrm{MgSO}_{4} ; 1 \mathrm{NaH}_{2} \mathrm{PO}_{4} ; 26.2$ $\mathrm{NaHCO}_{3}$; and 11 glucose, continuously bubbled with a mixture of $95 \% \mathrm{O}_{2} / 5 \% \mathrm{CO}_{2}$. Following recovery, slices were transferred to a recording chamber (Warner Instruments, Hamden, CT, United States) mounted on a fixed upright microscope (Olympus BX51, Olympus Co., Tokyo, Japan) and continuously perfused $(2-3 \mathrm{ml} / \mathrm{min})$ with ACSF, saturated with $95 \% \mathrm{O}_{2} / 5 \% \mathrm{CO}_{2}$ and heated to $30 \pm 1{ }^{\circ} \mathrm{C}$ using a solution heater (Warner Instruments).

\section{Electrophysiological Recordings}

The DRn neurons were visualized using the Olympus BX51 microscope equipped with a 40x water-immersion lens, differential interference contrast, and infrared optical filter. Somatic whole cell recordings were obtained from putative DRn 5-HT neurons with patch electrodes (3-5 m $\Omega$ ) filled with a solution containing (in $\mathrm{mM}$ ): 120 potassium gluconate; $10 \mathrm{KCl} ; 10 \quad \mathrm{Na}_{2}$-phosphocreatine; $10 \mathrm{HEPES}$; $1 \mathrm{MgCl}_{2} ; 1$ EGTA; 2 Na 2 -ATP; 0.25 Na-GTP (pH 7.3, osmolality 280-290 mOsm). The DRn 5-HT neurons were identified by their distinct electrical properties, which include slow firing activity induced by supra-threshold membrane depolarization, large after-hyperpolarization and membrane hyperpolarization induced by $5-\mathrm{HT}_{1 \mathrm{~A}}$ receptor agonist as previously described (Haj-Dahmane, 2001; Geddes et al., 2015). For the experiments assessing excitation/inhibition (E/I) ratio, whole cell recordings were performed with intracellular solution containing (in $\mathrm{mM}$ ) : 120 cesium methanesulfonate; $10 \mathrm{KCl} ; 10 \mathrm{Na}$-phosphocreatine; $10 \mathrm{HEPES} ; 1 \mathrm{MgCl}$; 1 EGTA; 2 Na2-ATP; 0.25 Na-GTP (pH 7.3, osmolality 280-290 mOsm).

All recordings were performed from putative 5-HT neurons located in the dorsomedial subdivision of the DRn. Excitatory postsynaptic currents (EPSCs) or inhibitory postsynaptic currents (IPSCs) were evoked with single square-pulses (duration $=100-200 \mu \mathrm{s}$ ) delivered at $0.1 \mathrm{~Hz}$ with patch pipettes (2-3 $\mathrm{m} \Omega$ ) filled with standard ACSF and placed $(50-100 \mu \mathrm{m})$ dorsolateral to the recording sites. In some experiments, to assess changes in paired-pulse ratio (PPR), pairs of EPSCs or IPSCs were evoked with an inter-stimulus interval (ISI) of 30-150 ms. The intensity of the stimulus was adjusted to evoke $75 \%$ of the maximal amplitude of EPSCs/IPSCs. The EPSCs were recorded from neurons voltage clamped at $-70 \mathrm{mV}$ in the presence of gamma-aminobutyric acid type $\mathrm{A}\left(\mathrm{GABA}_{\mathrm{A}}\right)$ and glycine receptor antagonists picrotoxin $(100 \mu \mathrm{M})$ and strychnine $(20 \mu \mathrm{M})$, respectively. $\mathrm{GABA}_{\mathrm{A}}$ receptor-mediated IPSCs were recorded from neurons voltage-clamped at $0 \mathrm{mV}$ in the presence of N-methyl-D-aspartic acid (NMDA) and AMPA receptor antagonists D-AP5 $(50 \mu \mathrm{M})$ and DNQX $(20 \mu \mathrm{M})$, respectively. To examine miniature action potentialindependent EPSCs (mEPSCs), tetrodotoxin $(1 \mu \mathrm{M})$ was added in the bath and mEPSCs were recorded for $150 \mathrm{~s}$. Membrane currents were amplified with an Axoclamp 2B or Multiclamp 700B amplifier (Molecular Devices, Union City, CA, United States). Membrane currents were filtered at $3 \mathrm{kHz}$, digitized at $20 \mathrm{kHz}$ with Digidata $1440 \mathrm{~A}$, and acquired using the pClamp 10 software (Molecular Devices). The cell input resistance and access resistance $(10-20 \mathrm{~m} \Omega$ ) were monitored throughout the experiments using $5 \mathrm{mV}$ hyperpolarizing voltage steps (500 ms duration). Recordings were discarded when the input and series resistance changed by more than $10-20 \%$.

To examine whether glutamatergic synapses onto DRn 5-HT neurons exhibit activity-dependent plasticity, we used a spiketiming-dependent long-term potentiation (tLTP) induction protocol that consisted of pairing a train of five bursts of presynaptic stimulation with back-propagating action potentials (bAPs) delivered at $5 \mathrm{~Hz}$. Each burst was composed of three presynaptic stimuli $(50 \mathrm{~Hz})$ paired with three bAPs $(50 \mathrm{~Hz})$ with a delay of +5 to $10 \mathrm{~ms}$. Action potentials were evoked by injection of depolarizing somatic current (1.5$2 \mathrm{nA}, 2 \mathrm{~ms}$ duration) in the current clamp mode. After obtaining a stable recording of EPSCs for at least $10 \mathrm{~min}$, the recordings of DRn 5-HT neurons were switched to the current clamp mode and a total of 20 trains were administered at $0.1 \mathrm{~Hz}$.

\section{Data Analyses}

The dependent variables of the behavioral tests were described above. For the electrophysiological recordings, the amplitudes of EPSCs and IPSCs were analyzed using Clampfit 10.2 software (Molecular Devices) and determined by measuring the average current during a $2 \mathrm{~ms}$ time window at the peak of each EPSC/IPSC, subtracted from the baseline current determined during a $5 \mathrm{~ms}$ time window before the stimulus artifact. The E/I ratio was defined as the ratio of the amplitudes of EPSCs and IPSCs obtained in the same neuron. The PPRs (EPSC2/EPSC1 or IPSC2/IPSC1) were averaged for at least 60 trials. For the analysis of tLTP, EPSC amplitudes were normalized to the mean baseline amplitude recorded for at least $10 \mathrm{~min}$ 

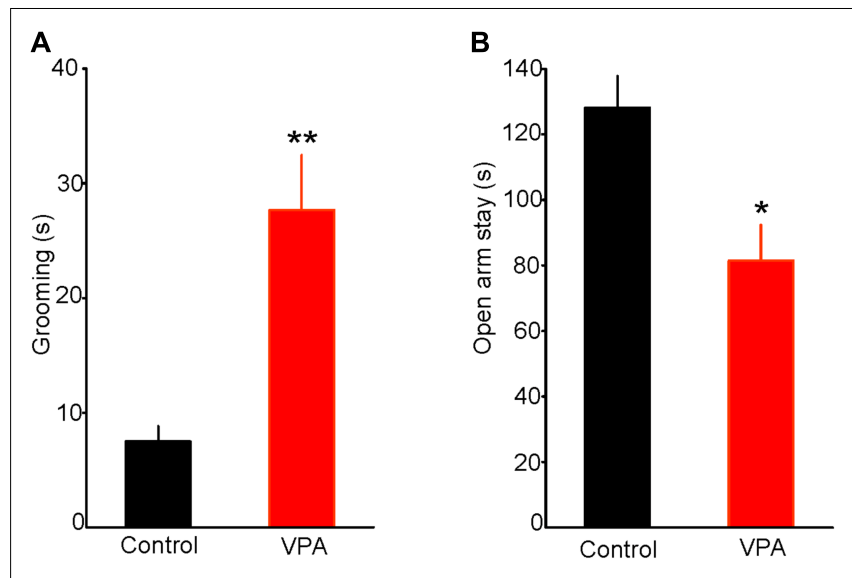

C

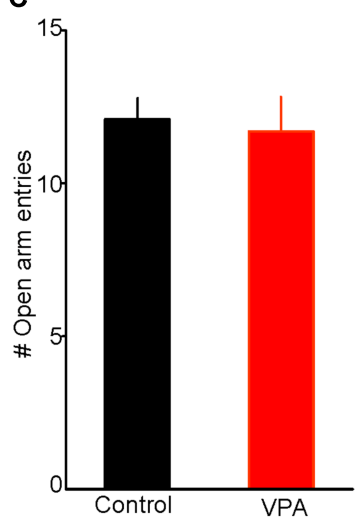

D

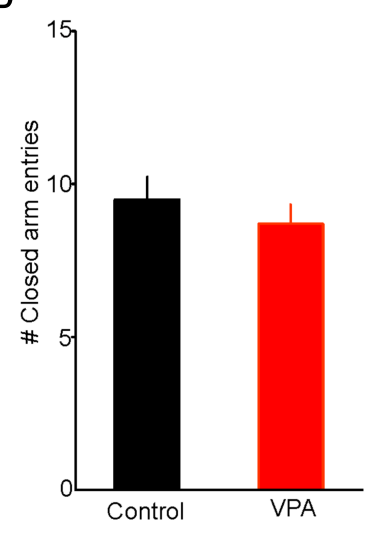

FIGURE 1 | Prenatal valproic acid (VPA) exposure increases anxiety-like behaviors. (A) Effect of prenatal VPA exposure on self-grooming. Note the significant increase in duration of self-grooming in VPA rats, indicating augmented anxiety (Control: $n=7$; VPA: $n=8, p<0.01$ ). (B) Effect of prenatal VPA exposure on the duration of open arm stay in the elevated plus maze. Note the significant reduction in open arm stay in VPA rats (Control: $n=10$; VPA: $n=7, p<0.05)$. (C,D) illustrate the effects of prenatal VPA exposure on the numbers of open and closed arm entries, respectively. Note that prenatal VPA exposure does not alter the numbers of entries into the open or closed arms (Control: $n=10$; VPA: $n=7 ; p>0.05$ for both). Data are presented as Mean \pm SEM. ${ }^{*} p<0.05 ;{ }^{* *} p<0.01$.

before the administration of the pairing protocol. Statistical analyses were performed, using Student's $t$ tests, analysis of variance (ANOVA), Kolmogorov-Smirnov tests (K-S tests), or Chi-square tests, with software SAS 9.4 (SAS Institute, Cary, NC, United States) or Origin 8.0 (MicroCal Software Inc., Northampton, MA, United States). Pairwise comparisons after ANOVA were conducted using post hoc Tukey tests. Statistical significance was set at $\alpha=0.05$. The results in the text and figures are expressed as mean \pm SEM.

\section{Chemicals}

Most chemicals were obtained from Fisher Scientific (Pittsburgh, PA, United States). VPA sodium salt and 6,7-dinitroquinoxaline2,3-dione (DNQX) were purchased from Sigma-Aldrich (Saint Louis, MO, United States). Picrotoxin, strychnine,

and D-(-)-2-Amino-5-phosphonopentanic acid (D-AP5) were obtained from Tocris Biosciences (Minneapolis, $\mathrm{MN}$, United States).

\section{RESULTS}

\section{Prenatal VPA Exposure Slightly Reduces Bodyweights on PD1 but Not Litter Sizes}

Fourteen control and 23 VPA female rats were used in breeding, among which 13 control and $20 \mathrm{VPA}$ exposed rats gave birth to live pups. A Chi-square test showed no significant group difference in proportion of litters with live pups. For number of live pups/litter, there was no significant group difference $($ Control $=9.54 \pm 0.95 ; \mathrm{VPA}=7.45 \pm 0.94)$, based on a two-way ANOVA (group: control vs. VPA; sex: male vs. female). In addition, prenatal VPA exposure resulted in slightly lower body weights on PD 1 in both male and female pups (Control Male: $7.05 \pm 0.11$ g; VPA Male: $6.47 \pm 0.10 \mathrm{~g}$; Control Female: $6.68 \pm 0.11 \mathrm{~g}$; VPA Female: $6.13 \pm 0.11 \mathrm{~g}$ ). A two-way nested ANOVA with litter as a nested variable produced a group main effect, $F_{1,246}=22.61, p<0.001$, a sex main effect, $F_{1,246}=9.83, p<0.01$, and a litter effect, $F_{23,246}=8.67, p<0.001$. Taken together, these results suggest that prenatal VPA treatment did not produce major teratogenic effects.

\section{Prenatal VPA Exposure Increases Stereotypy and Anxiety-Like Behaviors}

Results from previous animal studies have shown that prenatal VPA exposure induces a robust ASD-like phenotype, including repetitive behaviors (Lewis et al., 2007; Olexová et al., 2016; Nicolini and Fahnestock, 2018). Consistent with this notion, we found that a single injection of VPA (400 $\mathrm{mg} / \mathrm{kg}$; s.c.) on embryonic day 12.5 significantly increased self-grooming, a measure of stereotypy associated with augmented anxiety. Indeed, as illustrated in Figure 1A, VPA exposed rats spent more time engaging in self-grooming in a novel environment, compared with control rats (Control $=7.5 \pm 1.3 \mathrm{~s}, n=7$; $\mathrm{VPA}=27.8 \pm 5.5 \mathrm{~s}, n=8$; two-tailed $t$-test, $t_{13}=-3.34$, $p<0.01$, Figure 1A), thereby indicating that in our experimental condition, prenatal VPA exposure increases anxiety-like behavior.

We also tested the effect of prenatal VPA exposure on anxietylike behaviors using the EPM test. We found that prenatally VPA-exposed rats spent significantly less time on the open arms compared with controls (Controls $=125 \pm 9.6 \mathrm{~s}, n=10$; $\mathrm{VPA}=82 \pm 10.9, n=7 ; t_{15}=2.93, p<0.05$, Figure 1B). In contrast, prenatal VPA exposure did not significantly alter the numbers of entries into the open (Control $=12.1 \pm 0.7$; $\mathrm{VPA}=11.7 \pm 1.1 ; t_{15}=0.31, p=0.76$. Figure $\left.1 \mathrm{C}\right)$ or the closed arms (Control $=9.5 \pm 0.7 ; \mathrm{VPA}=8.7 \pm 0.6 ; t_{15}=0.75$, $p=0.47$, Figure 1D), indicating no significant effect on the rat locomotor activity in the EPM. Collectively, these results show that prenatal VPA exposure increases anxiety-like behavior in 

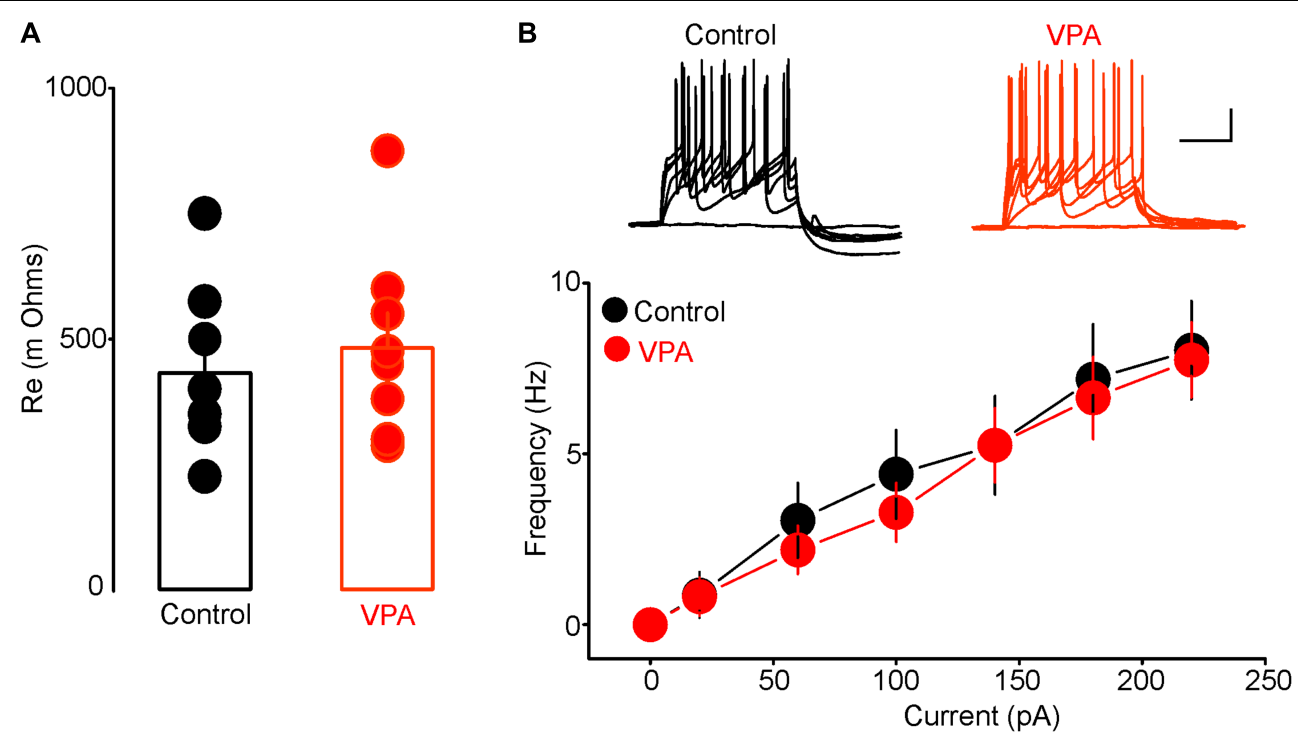

FIGURE 2 | Prenatal VPA exposure has no effect on the intrinsic excitability of dorsal raphe nucleus (DRn) 5-HT neurons. (A) Prenatal VPA exposure does not alter input resistance of DRn 5-HT neurons. (B) Prenatal VPA exposure does not alter the evoked firing activity of DRn 5-HT neurons. Upper panel illustrates the responses (number of action potentials) of DRn 5-HT neurons to depolarizing current pulses obtained in slices from control (left traces) and VPA exposed rats (right traces). Lower panel depicts the average input-output curves of responses obtained in control $(\bullet, n=8$ cells) and VPA rats (•, $n=8$ cells). Data are presented as Mean \pm SEM. Scale bars for the upper traces: $25 \mathrm{mV}$ (vertical), $200 \mathrm{~ms}$ (horizontal).

\section{$\mathbf{A}$}
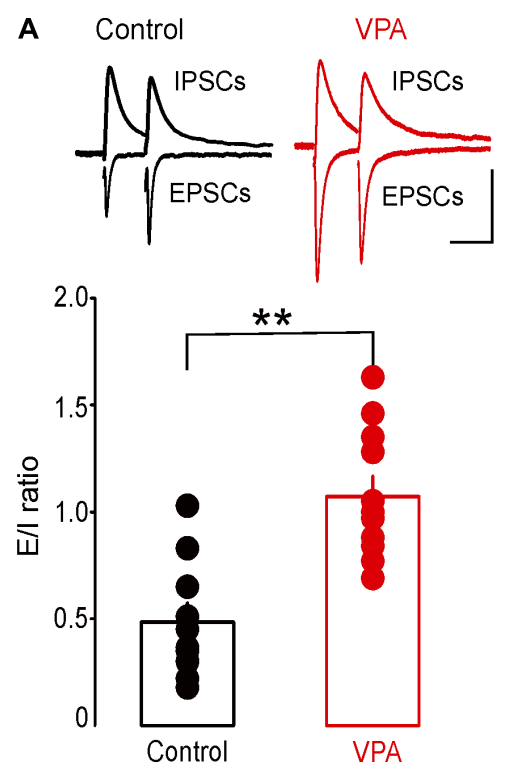

$* *$

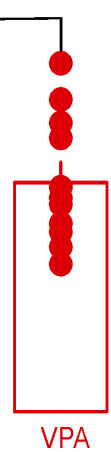

B
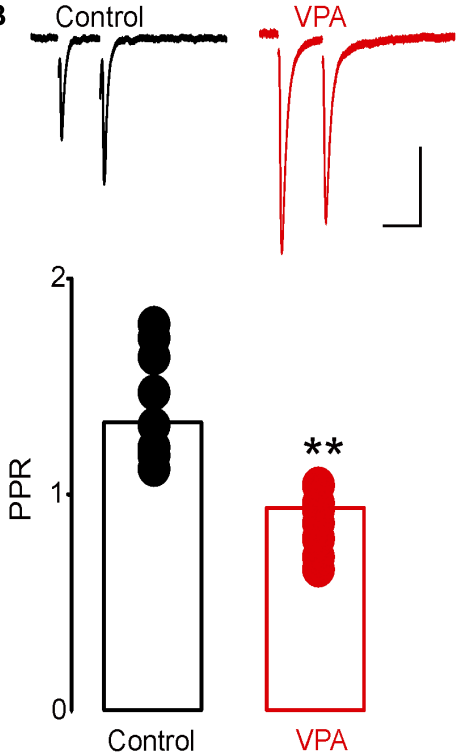

\section{C}
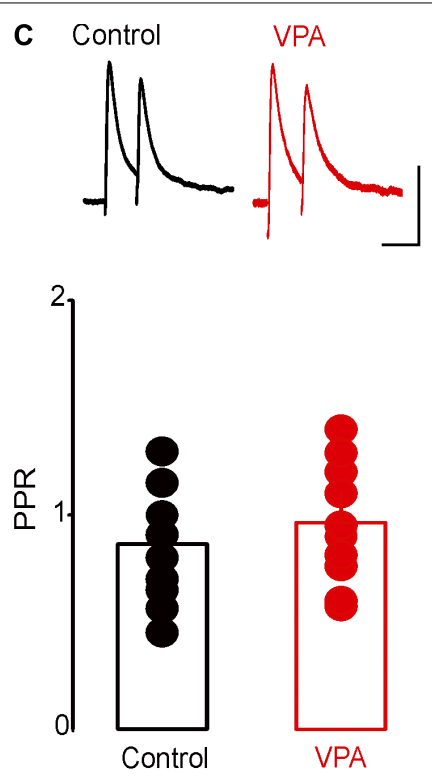

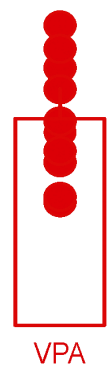

FIGURE 3 | Prenatal VPA exposure increases the synaptic excitation/inhibition (E/l) ratio by enhancing glutamatergic synaptic transmission. (A) Prenatal VPA exposure increases the synaptic E/I ratio. Upper panel depicts superimposed excitatory/inhibitory postsynaptic current (EPSC/IPSC) traces recorded from dorsal raphe nucleus 5-HT neurons in control (left traces) and VPA rats (right traces). Lower graph shows the average E/I ratio obtained in control and VPA rats. Note that prenatal VPA exposure significantly increases the E/I ratio (control: $n=10$ cells; VPA: $n=11$ cells, ${ }^{* *} p<0.01$ ). (B) Prenatal VPA exposure increases the probability of glutamate release. Upper panel illustrates superimposed EPSC traces induced by pairs of stimuli at $30 \mathrm{~ms}$ inter-stimulus interval (ISI) and recorded in slices from control (left traces) and VPA rats (right traces). Lower graph illustrates the average paired pulse ratios (PPR = EPSC2/EPSC1) obtained at $30 \mathrm{~ms}$ ISI in control (•, $n=10$ cells) and VPA rats ( $\bullet, n=9$ cells). Prenatal VPA exposure significantly reduces the PPRs of EPSCs at ISI $=30 \mathrm{~ms}(* * p<0.01)$. (C) Prenatal VPA exposure has no effect on the probability of GABA release. Upper graph illustrates superimposed pairs of GABAA-IPSCs recorded at $30 \mathrm{~ms}$ ISI in slices from control (left traces) and VPA rats (right traces). Lower graph is a summary of the average PPRs (IPSC2/IPSC1) of GABA-IPSCs obtained at $30 \mathrm{~ms}$ ISI in control ( $n=6 \mathrm{cells}$ ) and VPA rats ( $n=6$ cells). VPA exposure has no effect on the PPR of GABA-IPSCs at ISI $=30 \mathrm{~ms}(p>0.05)$. Data are presented as Mean \pm SEM. Scale bars for the upper traces: 100 pA (vertical), 20 ms (horizontal). 
the EPM and that this anxiety phenotype is not associated with altered locomotor activity.

\section{Prenatal VPA Exposure Enhances the Activity of Putative DRn 5-HT Neurons}

To determine whether the increased anxiety-like behaviors in rats with prenatal VPA exposure were associated with alterations in excitability of putative DRn 5-HT neurons, we performed conventional ex vivo whole-cell recordings and assessed the impact of prenatal VPA exposure on the resting membrane potential and intrinsic excitability of these neurons. As reported previously (Vandermaelen and Aghajanian, 1983; Williams et al., 1988; Haj-Dahmane, 2001), in slices from control rats, putative DRn 5-HT neurons were quiescent and exhibited a hyperpolarized resting membrane potential (Control: $-64.54 \pm 2.8 \mathrm{mV}, n=16$ cells). Remarkably, in slices from VPA exposed rats, all putative DRn 5-HT neurons recorded were depolarized and exhibited spontaneously firing activity (Frequency $=3.2 \pm 1.5 \mathrm{~Hz} ; n=18$ cells). These results indicate that prenatal VPA exposure increases the activity of putative DRn 5-HT neurons. In order to determine the cellular mechanisms underlying this effect, we assessed the impact of prenatal VPA exposure on the membrane input resistance and evoked firing activity of DRn 5-HT neurons. Our results showed that prenatal VPA exposure did not significantly alter the membrane resistance (Control $=481 \pm 70 \mathrm{~m} \Omega, n=8$ cells; $\mathrm{VPA}=431 \pm 60 \mathrm{~m} \Omega, n=8$ cells, $p>0.05$, Figure $2 \mathrm{~A}$ ) or evoked activity of DRn 5-HT neurons (Figure 2B). Taken together, these results indicate that the increased activity of DRn 5-HT neurons observed in rats with prenatal VPA exposure is unlikely to be mediated by alterations in their intrinsic excitability, but most likely by a persistent change in excitatory and/or inhibitory inputs.

\section{Prenatal VPA Exposure Enhances Glutamatergic Synaptic Transmission in the DRn}

To test the effect of prenatal VPA exposure on excitatory and inhibitory synaptic transmission onto putative DRn 5HT neurons, we first assessed the E/I ratio, a measure of overall synaptic network activity in slices from control and VPA exposed rats. We found that prenatal VPA exposure significantly increased the $\mathrm{E} / \mathrm{I}$ ratio (Control: $\mathrm{E} / \mathrm{I}=0.48 \pm 0.08$, $n=10$ cells; VPA: $\mathrm{E} / \mathrm{I}=1.07 \pm 0.09, n=11$ cells, $p<0.01$, Figure 3A). We next examined the effects of prenatal VPA exposure on the probability of glutamate and GABA release, assessed by PPRs of EPSCs and $\mathrm{GABA}_{\mathrm{A}}$-IPSCs, respectively. The results of these experiments showed that prenatal VPA exposure significantly reduced the PPRs of EPSCs determined at ISI $=30$ and $50 \mathrm{~ms}$ (Control: PPR $(30 \mathrm{~ms})=1.28 \pm 0.09$, $n=10$ cells; VPA: PPR $(30 \mathrm{~ms})=1.01 \pm 0.04, n=11$ cells, $p<0.01$, Figure 3B). In contrast, prenatal VPA exposure did not significantly affect the PPRs of $\mathrm{GABA}_{\mathrm{A}}$-IPSCs (Control: $\mathrm{PPR}(30 \mathrm{~ms})=0.85 \pm 0.09, n=6$ cells; VPA: PPR $(30 \mathrm{~ms})=0.92 \pm 0.14, n=6$ cells, $p>0.05$, Figure $3 \mathrm{C})$. Collectively, these results indicate that prenatal VPA exposure potentiates the probability of glutamate but not GABA release, which contributes to the increased E/I ratio in DRn 5-HT neurons.

To further test the effect of prenatal VPA exposure on glutamate release, we examined the frequency and amplitude of mEPSCs, a measure of quantal glutamate release. The mEPSCs were recorded from putative DRn 5-HT neurons in slices from control and VPA exposed rats. The results of this experiment showed that prenatal VPA exposure profoundly increased the frequency of mEPSCs (Figure 4A), which induced a significant leftward shift of the cumulative distribution of

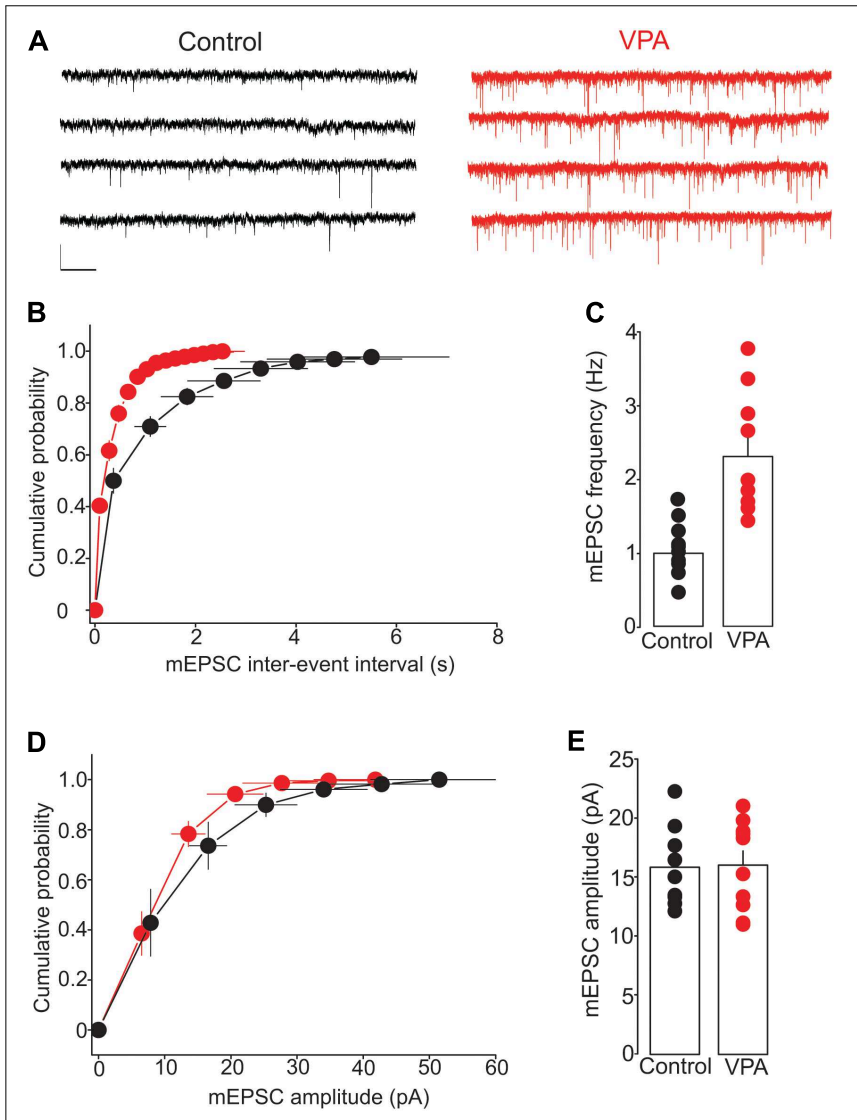

FIGURE 4 | Prenatal VPA exposure increases the frequency but not the amplitude of miniature excitatory postsynaptic currents (mEPSCs). (A) Illustrates current traces recorded from dorsal raphe nucleus 5-HT neurons in slices from control (left traces) and VPA rats (right traces). (B) Represents the average cumulative distribution of mEPSC inter-event intervals obtained in control $(\bullet, n=8$ cells) and VPA rats $(\bullet, n=9$ cells). Prenatal VPA exposure induces a significant leftward shift of the cumulative distribution of the inter-event intervals of mEPSCs ( $p<0.05$, Kolmogorov-Smirnov test). (C) Depicts the average mEPSC frequencies obtained in control and VPA rats, which indicates a significant increase in MEPSC frequency in VPA rats compared with controls $(* * p<0.01)$. (D) Illustrates the average cumulative distribution of mEPSC amplitudes recorded in control and VPA rats.

(E) Represents the average amplitudes of mEPSCs recorded in control and VPA rats. Note that prenatal VPA exposure has no significant effect on the cumulative distribution $(p>0.05$, Kolmogorov-Smirnov test) of or the average mEPSC amplitude $(p>0.05)$. Data are presented as Mean \pm SEM. Scale bars in (A): $10 \mathrm{pA}$ (vertical), $1 \mathrm{~s}$ (horizontal). 
mEPSC inter-event interval (Control: $n=8$ cells; VPA: $n=9$ cells, $p<0.05, \mathrm{~K}-\mathrm{S}$ test, Figure 4B). Indeed, the average mEPCS frequency was increased from $0.98 \pm 0.1 \mathrm{~Hz}$ in controls to $2.23 \pm 0.25 \mathrm{~Hz}$ in VPA exposed rats $(p<0.05$, Figure 4C). In contrast, prenatal VPA exposure did not significantly alter the cumulative distribution (Figure 4D) of or the average mEPSC amplitude (Control $=15.9 \pm 1.1 \mathrm{pA}$; $\mathrm{VPA}=16.1 \pm 1.2 \mathrm{pA}, p>0.05$, Figure 4E). Taken together, these results indicate that prenatal VPA exposure persistently increases glutamate release at glutamatergic synapses onto DRn 5-HT neurons.

\section{Prenatal VPA Exposure Occludes the tLTP of Glutamatergic Synapses in the DRn}

Results from a previous study have shown that glutamatergic synapses onto DRn 5-HT neurons undergo tLTP. Thus, pairing presynaptic stimulation with postsynaptic action potentials elicits tLTP of AMPR-EPSCs. This form of synaptic plasticity is mediated by an increase in glutamate release (Haj-Dahmane et al., 2017). Because prenatal VPA exposure persistently increases the probability of glutamate release, we examined whether this effect could occlude the tLTP in DRn 5-HT neurons. To that end, we assessed the impact of prenatal VPA exposure on the induction and magnitude of the tLTP. We found that the pairing protocol, which reliably induced tLTP in DRn 5-HT neurons of control rats, failed to induce tLTP in prenatally VPA-exposed rats (tLTP Control $=145.1 \pm 10 \%$ of baseline, $n=10$ cells; tLTP VPA $=104.8 \pm 7.3 \%$ of baseline, $n=10$ cells, $p<0.05$, Figure 5). These results suggest that the persistent increase in glutamate release induced by prenatal VPA exposure occludes the tLTP.

\section{DISCUSSION}

Abnormal functions of the central 5-HT system are involved in several neurodevelopmental disorders including ASD. However, it remains unknown whether the function of DRn 5-HT neurons is impaired in ASD. In the present study, we show that increased anxiety and stereotypy in the VPA rat model of ASD are associated with an enhanced firing activity of putative DRn 5-HT neurons. Importantly, our results also reveal that this effect could be attributed to a persistent potentiation of glutamatergic synaptic transmission. As such, these results identify a potential cellular mechanism by which prenatal VPA exposure alters the function of the 5HT system, which may underlie the behavioral phenotypes observed in the VPA model of ASD. The finding also provides potential cellular mechanisms mediating the pathophysiology of ASD.

Prenatal VPA exposure is a well-established environmental risk factor for the development of ASD (Christensen et al., 2013; Roullet et al., 2013; Ornoy et al., 2015). Results from numerous studies in mice and rats have shown that a single injection of VPA around embryonic day 12.5 to pregnant dams elicits ASD-like phenotypes, which include impaired social and communicative deficits and increased stereotypy (Favre et al., 2013; Roullet et al., 2013; Nicolini and Fahnestock, 2018). In addition to these core symptoms, prenatal VPA exposure also induces alterations in stress homeostasis and increases anxiety-like behaviors (Banerjee et al., 2014; Olexová et al., 2016). Consistent with these observations, results from the present study show that prenatal VPA exposure increases anxiety-like behaviors. Importantly, the behavioral phenotype is associated with physiological alterations in the DRn 5-HT system.

Previous studies examining the impact of prenatal VPA exposure on the central 5-HT system have largely focused on the effects of VPA on the early development of 5-HT neurons (Narita et al., 2002; Miyazaki et al., 2005; Tsujino et al., 2007). Generally, these studies have shown that, when administered on embryonic days 9 - 12 (neural plate stage), VPA irreversibly alters the differentiation and migration of 5-HT neurons to the DRn (Narita et al., 2002; Tsujino et al., 2007). In addition, prenatal VPA exposure has been shown to increase 5-HT levels in the forebrain areas (Narita et al., 2002; Tsujino et al., 2007), suggesting an enhanced 5-HT neurotransmission in VPA exposed rats. Results from the present study reveal for the first time, that prenatal VPA exposure persistently increases the firing activity of DRn 5-HT neurons. This observation provides a cellular mechanism by which prenatal VPA exposure enhances central 5-HT transmission. Importantly, because hyperserotonemia is thought to mediate increased anxiety, our findings provide a potential neuronal mechanism for behavioral dysfunctions in ASD.

The results from the present study show that the increased firing activity of DRn 5-HT neurons in rats with prenatal VPA exposure may be mediated by alterations in their synaptic inputs. Indeed, we have shown that prenatal VPA exposure persistently increases the ratio of synaptic E/I in DRn 5-HT neurons. Interestingly, an increase in synaptic $\mathrm{E} / \mathrm{I}$ ratio has also been reported in other brain areas of prenatally VPA exposed rats (Kim et al., 2013; Lin et al., 2013) and numerous genetic models of ASD (Rubenstein and Merzenich, 2003; Dani et al., 2005; Lee et al., 2015). Collectively, these studies indicate that an imbalance of synaptic E/I is a common effect of both environmental and genetic risk factors for ASD, which could play a major role in the pathophysiology of ASD (Yizhar et al., 2011; Lee et al., 2017).

The results from the present study show that the increased E/I ratio in DRn 5-HT neurons of VPA exposed rats is mainly mediated by a persistent potentiation of glutamate release. This conclusion is supported by the observation that prenatal VPA exposure decreases the PPR of EPSCs and increases the frequency of mEPSCs, but has no significant effect on GABAergic synaptic transmission. These results are consistent with previous studies reporting increased glutamate release in other brain areas of VPA exposed rats (Markram et al., 2008; Lin et al., 2013). Such results support the notion that an enhanced glutamatergic transmission is a common mechanism underlying the synaptic E/I imbalance observed in the VPA model of ASD. In contrast, studies using various genetic models of ASD have reported that alterations in both glutamate and GABA concentrations and/or synaptic 
transmission contribute to the imbalance of synaptic E/I (Lee et al., 2017). For example, BTBR mice with ASD behavioral phenotypes have both increased glutamate and decreased GABA concentrations in the prefrontal cortex (PFC) and amygdala (Bove et al., 2018).

Prenatal VPA administration has also been shown to alter synaptic plasticity. Indeed, VPA exposed rats exhibit an enhanced NMDA receptor function (Rinaldi et al., 2007; Kang and Kim, 2015) and NMDA-mediated LTP of glutamatergic synapses in the lateral amygdala (Markram et al., 2008; Lin et al., 2013) and the medial PFC (Rinaldi et al., 2008; Sui and Chen, 2012). We show that in DRn 5-HT neurons, while glutamatergic transmission is enhanced in VPA exposed rats, the tLTP of glutamatergic synapses onto 5-HT neurons is impaired. Because the tLTP in DRn 5-HT neurons is mediated by presynaptic increase in glutamate release and prenatal VPA exposure persistently increases glutamate release, the inhibition of tLTP observed in VPA exposed rats is most likely mediated by an occlusion effect. These observations suggest that the glutamatergic synaptic strength is maximized and the normal function of synaptic homeostasis is impaired in DRn 5-HT neurons of VPA rats. Such an effect could further contribute to behavioral deficits associated with 5-HT function (Daly et al., 2014).

We have previously shown that the tLTP of glutamatergic synapses onto DRn 5-HT neurons is mediated by the activation of nitric oxide (NO) and the protein kinase $\mathrm{G}$ signaling pathways (Haj-Dahmane et al., 2017). Interestingly, results from numerous studies have shown that prenatal VPA exposure promotes oxidative stress and enhances the formation of nitric oxide (Frustaci et al., 2012; Mario Tiboni and Ponzano, 2014; Yui et al., 2016). These effects are mediated by an increased function and expression of neuronal nitric oxide synthase (nNOS) (Mario Tiboni and Ponzano, 2014). Thus, it is tempting to speculate that the increased glutamate release and occlusion of the tLTP in DRn 5-HT neurons of VPA exposed rats could be mediated by enhanced NO signaling. However, future studies are required to directly confirm this hypothesis.

Taken together, results from the present study show that a major phenotype of ASD, increased anxiety-like behaviors, is observed after prenatal VPA exposure, a well-characterized nongenetic model of ASD. Importantly, we show increased DRn 5HT firing caused by enhanced glutamatergic neurotransmission

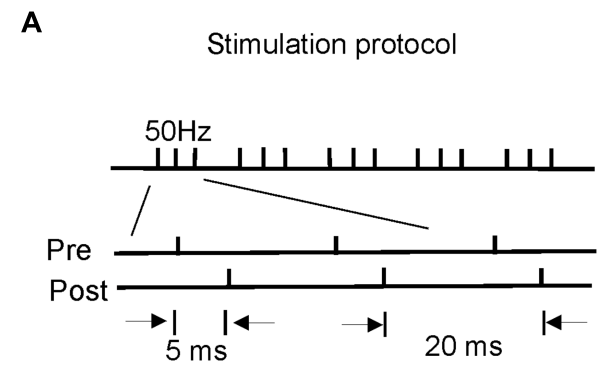

B

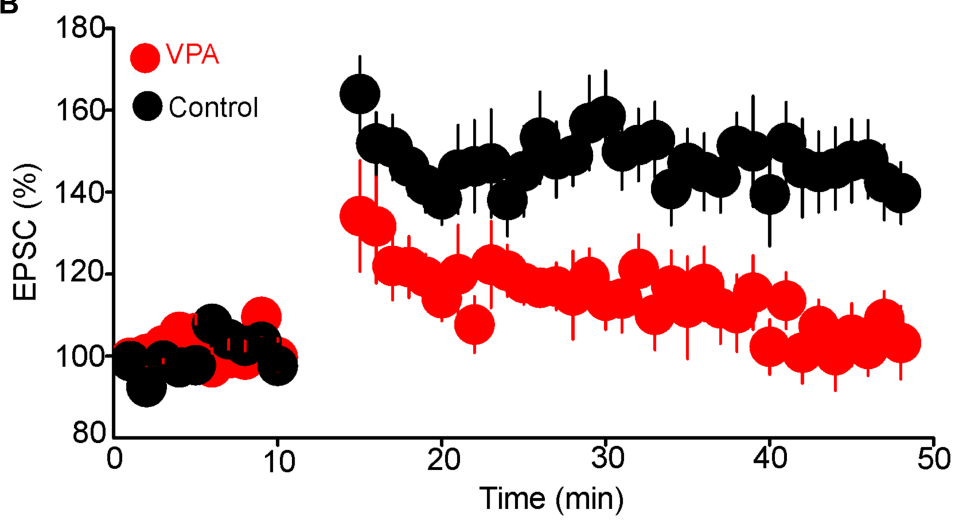

Response

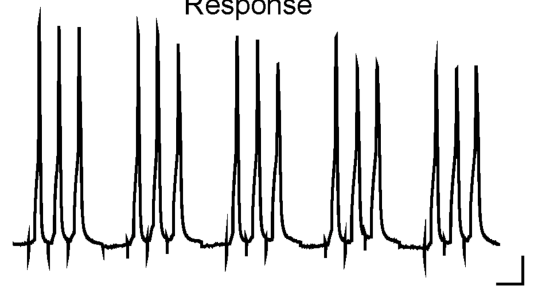

FIGURE 5 | Prenatal VPA exposure occludes the spike-timing-dependent long-term potentiation (tLTP) of glutamatergic synapses in the dorsal raphe nucleus (DRn). (A) Depicts the stimulation protocol (left traces) and the responses (right traces) during the tLTP induction. Scale bars for the traces: $25 \mathrm{mV}$ (vertical), $10 \mathrm{~ms}$ (horizontal). (B) Lower graph illustrates the time course of the tLTP in DRn slices from control $(\bullet)$ and VPA rats $(\bullet)$. Note that pairing presynaptic stimulation with back-propagating action potentials induces a robust tLTP in control (140.5 $\pm 5.6 \%$ of baseline, $n=10$ cells, $p<0.01$ versus baseline), but not in VPA rats (105.4 $\pm 3.6 \%$ of baseline, $n=10$ cells, $p>0.05$ versus baseline). Right traces are superimposed excitatory postsynaptic currents recorded pre- and post-application of the tLTP induction protocol in slices from control (upper traces) and VPA rats (lower traces). Data are presented as Mean \pm SEM. Scale bars for the traces: $50 \mathrm{pA}$ (vertical), $20 \mathrm{~ms}$ (horizontal). 
in VPA exposed rats. This effect is accompanied by impaired plasticity in glutamatergic synapses. It is well established that the DRn 5-HT system plays an important role in mediating anxietylike behaviors (Iversen, 1984; Graeff et al., 1996). Therefore, our results provide potential cellular mechanisms for these behaviors associated with hyperserotonemia in individuals with ASD.

\section{AUTHOR CONTRIBUTIONS}

RW conducted animal breeding and performed the behavioral experiments. $\mathrm{KH}$ and R-YS conducted prenatal animal treatment,

\section{REFERENCES}

Abrahams, B. S., Arking, D. E., Campbell, D. B., Mefford, H. C., Morrow, E. M., Weiss, L. A., et al. (2013). SFARI Gene 2.0: a community-driven knowledgebase for the autism spectrum disorders (ASDs). Mol. Autism 4:36. doi: 10.1186/20402392-4-36

Abrahams, B. S., and Geschwind, D. H. (2008). Advances in autism genetics: on the threshold of a new neurobiology. Nat. Rev. Genet. 9, 341-355. doi: 10.1038/ $\operatorname{nrg} 2346$

American Psychiatric Association (2013). Diagnostic and Statistical Manual of Mental Disorders (DSM- $\left.5^{\circledR}\right)$. Washington, DC: American Psychiatric Pub. doi: 10.1176/appi.books.9780890425596

Arieff, Z., Kaur, M., Gameeldien, H., Van Der Merwe, L., and Bajic, V. B. (2010). 5-HTTLPR polymorphism: analysis in South African autistic individuals. Hum. Biol. 82, 291-300. doi: 10.3378/027.082.0303

Banerjee, A., Engineer, C. T., Sauls, B. L., Morales, A. A., Kilgard, M. P., and Ploski, J. E. (2014). Abnormal emotional learning in a rat model of autism exposed to valproic acid in utero. Front. Behav. Neurosci. 8:387. doi: 10.3389/fnbeh.2014. 00387

Bove, M., Ike, K., Eldering, A., Buwalda, B., De Boer, S. F., Morgese, M. G., et al. (2018). The visible burrow system: a behavioral paradigm to assess sociability and social withdrawal in BTBR and C57BL/6J mice strains. Behav. Brain Res. 344, 9-19. doi: 10.1016/j.bbr.2018.02.003

Christensen, J., Grønborg, T. K., Sørensen, M. J., Schendel, D., Parner, E. T., Pedersen, L. H., et al. (2013). Prenatal valproate exposure and risk of autism spectrum disorders and childhood autism. JAMA 309, 1696-1703. doi: 10.1001/ jama.2013.2270

Cook, E. H. Jr., Charak, D. A., Arida, J., Spohn, J. A., Roizen, N. J. M., and Leventhal, B. L. (1994). Depressive and obsessive-compulsive symptoms in hyperserotonemic parents of children with autistic disorder. Psychiatry Res. 52, 25-33. doi: 10.1016/0165-1781(94)90117-1

Dahlström, A., and Fuxe, K. (1964). Localization of monoamines in the lower brain stem. Cell. Mol. Life Sci. 20, 398-399. doi: 10.1007/BF02147990

Daly, E., Ecker, C., Hallahan, B., Deeley, Q., Craig, M., Murphy, C., et al. (2014). Response inhibition and serotonin in autism: a functional MRI study using acute tryptophan depletion. Brain 137, 2600-2610. doi: 10.1093/brain/awu178

Dani, V. S., Chang, Q., Maffei, A., Turrigiano, G. G., Jaenisch, R., and Nelson, S. B. (2005). Reduced cortical activity due to a shift in the balance between excitation and inhibition in a mouse model of Rett syndrome. Proc. Natl. Acad. Sci. U.S.A. 102, 12560-12565. doi: 10.1073/pnas.0506071102

Descarries, L., Watkins, K. C., Garcia, S., and Beaudet, A. (1982). The serotonin neurons in nucleus raphe dorsalis of adult rat: a light and electron microscope radioautographic study. J. Comp. Neurol. 207, 239-254. doi: 10.1002/cne. 902070305

Devlin, B., Cook, E., Coon, H., Dawson, G., Grigorenko, E., Mcmahon, W., et al. (2005). Autism and the serotonin transporter: the long and short of it. Mol. Psychiatry 10, 1110-1116. doi: 10.1038/sj.mp.4001724

Favre, M. R., Barkat, T. R., Mendola, D. L., Khazen, G., Markram, H., and Markram, K. (2013). General developmental health in the VPA-rat model of autism. Front. Behav. Neurosci. 7:88. doi: 10.3389/fnbeh.2013.00088

Frustaci, A., Neri, M., Cesario, A., Adams, J. B., Domenici, E., Dalla Bernardina, B., et al. (2012). Oxidative stress-related biomarkers in autism: systematic review participated in the experimental design, and coordinated the study. SH-D directed the project and performed the electrophysiological experiments. SH-D, RW, and R-YS wrote the manuscript.

\section{FUNDING}

This work was supported by the Howard T. Blane Director's Award for Development of Innovative Research in the Addictions of the Research Institute on Addictions, University at Buffalo, The State University of New York (BDAA Award to SH-D).

and meta-analyses. Free Radic. Biol. Med. 52, 2128-2141. doi: 10.1016/j. freeradbiomed.2012.03.011

Gabriele, S., Sacco, R., and Persico, A. M. (2014). Blood serotonin levels in autism spectrum disorder: a systematic review and meta-analysis. Eur. Neuropsychopharmacol. 24, 919-929. doi: 10.1016/j.euroneuro.2014.02.004

Geddes, S. D., Assadzada, S., Sokolovski, A., Bergeron, R., Haj-Dahmane, S., and Béique, J.-C. (2015). Time-dependent modulation of glutamate synapses onto 5-HT neurons by antidepressant treatment. Neuropharmacology 95, 130-143. doi: 10.1016/j.neuropharm.2015.02.027

Gispen, W., and Isaacson, R. (1981). ACTH-induced excessive grooming in the rat. Pharmacol. Ther. 12, 209-246. doi: 10.1016/0163-7258(81)90081-4

Gobbi, G., Bambico, F. R., Mangieri, R., Bortolato, M., Campolongo, P., Solinas, M., et al. (2005). Antidepressant-like activity and modulation of brain monoaminergic transmission by blockade of anandamide hydrolysis. Proc. Natl. Acad. Sci. U.S.A. 102, 18620-18625. doi: 10.1073/pnas.050959 1102

Grabrucker, A. M. (2013). Environmental factors in autism. Front. Psychiatry 3:118. doi: 10.3389/fpsyt.2012.00118

Graeff, F. G., Guimarães, F. S., De Andrade, T. G., and Deakin, J. F. (1996). Role of 5-HT in stress, anxiety, and depression. Pharmacol. Biochem. Behav. 54, 129-141. doi: 10.1016/0091-3057(95)02135-3

Haj-Dahmane, S. (2001). D2-like dopamine receptor activation excites rat dorsal raphe 5-HT neurons in vitro. Eur. J. Neurosci. 14, 125-134. doi: 10.1046/j.0953816x.2001.01616.x

Haj-Dahmane, S., Béique, J. C., and Shen, R.-Y. (2017). GluA2-Lacking AMPA receptors and nitric oxide signaling gate spike-timing-dependent potentiation of glutamate synapses in the dorsal raphe nucleus. eNeuro 4:ENEURO.011617.2017. doi: 10.1523/ENEURO.0116-17.2017

Hogg, S. (1996). A review of the validity and variability of the elevated plusmaze as an animal model of anxiety. Pharmacol. Biochem. Behav. 54, 21-30. doi: 10.1016/0091-3057(95)02126-4

Huang, C. H., and Santangelo, S. L. (2008). Autism and serotonin transporter gene polymorphisms: a systematic review and meta-analysis. Am. J. Med. Genet. Part B Neuropsychiatr. Genet. 147, 903-913. doi: 10.1002/ajmg.b.30720

Iversen, S. D. (1984). 5-HT and anxiety. Neuropharmacology 23, 1553-1560. doi: 10.1016/0028-3908(84)90099-6

Kalueff, A. V., and Tuohimaa, P. (2005). Mouse grooming microstructure is a reliable anxiety marker bidirectionally sensitive to GABAergic drugs. Eur. J. Pharmacol. 508, 147-153. doi: 10.1016/j.ejphar.2004.11.054

Kang, J., and Kim, E. (2015). Suppression of NMDA receptor function in mice prenatally exposed to valproic acid improves social deficits and repetitive behaviors. Front. Mol. Neurosci. 8:17. doi: 10.3389/fnmol.2015.00017

Kim, K. C., Kim, P., Go, H. S., Choi, C. S., Park, J. H., Kim, H. J., et al. (2013). Male-specific alteration in excitatory post-synaptic development and social interaction in pre-natal valproic acid exposure model of autism spectrum disorder. J. Neurochem. 124, 832-843. doi: 10.1111/jnc.12147

Landrigan, P. J. (2010). What causes autism? Exploring the environmental contribution. Curr. Opin. Pediatr. 22, 219-225. doi: 10.1097/MOP. 0b013e328336eb9a

Lee, E., Lee, J., and Kim, E. (2017). Excitation/inhibition imbalance in animal models of autism spectrum disorders. Biol. Psychiatry 81, 838-847. doi: 10.1016/ j.biopsych.2016.05.011 
Lee, J., Chung, C., Ha, S., Lee, D., Kim, D.-Y., Kim, H., et al. (2015). Shank3-mutant mice lacking exon 9 show altered excitation/inhibition balance, enhanced rearing, and spatial memory deficit. Front. Cell. Neurosci. 9:94. doi: 10.3389/ fncel.2015.00094

Lewis, M. H., Tanimura, Y., Lee, L. W., and Bodfish, J. W. (2007). Animal models of restricted repetitive behavior in autism. Behav. Brain Res. 176, 66-74. doi: 10.1016/j.bbr.2006.08.023

Lin, H.-C., Gean, P.-W., Wang, C.-C., Chan, Y.-H., and Chen, P. S. (2013). The amygdala excitatory/inhibitory balance in a valproate-induced rat autism model. PLoS One 8:e55248. doi: 10.1371/journal.pone.0055248

Mario Tiboni, G., and Ponzano, A. (2014). Nitric oxide and teratogenesis: an update. Curr. Pharm. Des. 20, 5443-5447. doi: 10.2174/ 1381612820666140205150437

Markram, K., Rinaldi, T., La Mendola, D., Sandi, C., and Markram, H. (2008). Abnormal fear conditioning and amygdala processing in an animal model of autism. Neuropsychopharmacology 33, 901-912. doi: 10.1038/sj.npp.1301453

Miyazaki, K., Narita, N., and Narita, M. (2005). Maternal administration of thalidomide or valproic acid causes abnormal serotonergic neurons in the offspring: implication for pathogenesis of autism. Int. J. Dev. Neurosci. 23, 287-297. doi: 10.1016/j.ijdevneu.2004.05.004

Muller, C. L., Anacker, A. M., and Veenstra-Vanderweele, J. (2016). The serotonin system in autism spectrum disorder: from biomarker to animal models. Neuroscience 321, 24-41. doi: 10.1016/j.neuroscience.2015.11.010

Narita, N., Kato, M., Tazoe, M., Miyazaki, K., Narita, M., and Okado, N. (2002). Increased monoamine concentration in the brain and blood of fetal thalidomide-and valproic acid-exposed rat: putative animal models for autism. Pediatr. Res. 52, 576-579.

Nicolini, C., and Fahnestock, M. (2018). The valproic acid-induced rodent model of autism. Exp. Neurol. 299, 217-227. doi: 10.1016/j.expneurol.2017. 04.017

Olexová, L., Štefánik, P., and Kršková, L. (2016). Increased anxiety-like behaviour and altered GABAergic system in the amygdala and cerebellum of VPA ratsAn animal model of autism. Neurosci. Lett. 629, 9-14. doi: 10.1016/j.neulet. 2016.06.035

Ornoy, A., Weinstein-Fudim, L., and Ergaz, Z. (2015). Prenatal factors associated with autism spectrum disorder (ASD). Reprod. Toxicol. 56, 155-169. doi: 10. 1016/j.reprotox.2015.05.007

Rinaldi, T., Kulangara, K., Antoniello, K., and Markram, H. (2007). Elevated NMDA receptor levels and enhanced postsynaptic long-term potentiation induced by prenatal exposure to valproic acid. Proc. Natl. Acad. Sci. U.S.A. 104, 13501-13506. doi: 10.1073/pnas.0704391104

Rinaldi, T., Perrodin, C., and Markram, H. (2008). Hyper-connectivity and hyperplasticity in the medial prefrontal cortex in the valproic acid animal model of autism. Front. Neural Circuits 2:4. doi: 10.3389/neuro.04.004.2008

Rodgers, R., and Dalvi, A. (1997). Anxiety, defence and the elevated plusmaze. Neurosci. Biobehav. Rev. 21, 801-810. doi: 10.1016/S0149-7634(96)00 058-9

Roullet, F. I., Lai, J. K., and Foster, J. A. (2013). In utero exposure to valproic acid and autism - a current review of clinical and animal studies. Neurotoxicol. Teratol. 36, 47-56. doi: 10.1016/j.ntt.2013.01.004
Rubenstein, J., and Merzenich, M. M. (2003). Model of autism: increased ratio of excitation/inhibition in key neural systems. Genes Brain Behav. 2, 255-267. doi: 10.1034/j.1601-183X.2003.00037.x

Schain, R. J., and Freedman, D. X. (1961). Studies on 5-hydroxyindole metabolism in autistic and other mentally retarded children. J. Pediatr. 58, 315-320. doi: 10.1016/S0022-3476(61)80261-8

Schneider, T., and Przewłocki, R. (2005). Behavioral alterations in rats prenatally exposed to valproic acid: animal model of autism. Neuropsychopharmacology 30, 80-89. doi: 10.1038/sj.npp.1300518

Sui, L., and Chen, M. (2012). Prenatal exposure to valproic acid enhances synaptic plasticity in the medial prefrontal cortex and fear memories. Brain Res. Bull. 87, 556-563. doi: 10.1016/j.brainresbull.2012.01.011

Tick, B., Bolton, P., Happé, F., Rutter, M., and Rijsdijk, F. (2016). Heritability of autism spectrum disorders: a meta-analysis of twin studies. J. Child Psychol. Psychiatry 57, 585-595. doi: 10.1111/jcpp.12499

Tsujino, N., Nakatani, Y., Seki, Y., Nakasato, A., Nakamura, M., Sugawara, M., et al. (2007). Abnormality of circadian rhythm accompanied by an increase in frontal cortex serotonin in animal model of autism. Neurosci. Res. 57, 289-295. doi: 10.1016/j.neures.2006.10.018

Vandermaelen, C., and Aghajanian, G. (1983). Electrophysiological and pharmacological characterization of serotonergic dorsal raphe neurons recorded extracellularly and intracellularly in rat brain slices. Brain Res. 289, 109-119. doi: 10.1016/0006-8993(83)90011-2

White, S. W., Oswald, D., Ollendick, T., and Scahill, L. (2009). Anxiety in children and adolescents with autism spectrum disorders. Clin. Psychol. Rev. 29, 216-229. doi: 10.1016/j.cpr.2009.01.003

Williams, J., Colmers, W., and Pan, Z. (1988). Voltage-and ligandactivated inwardly rectifying currents in dorsal raphe neurons in vitro. J. Neurosci. 8, 3499-3506. doi: 10.1523/JNEUROSCI.08-09-03499. 1988

Yizhar, O., Fenno, L. E., Prigge, M., Schneider, F., Davidson, T. J., O’shea, D. J., et al. (2011). Neocortical excitation/inhibition balance in information processing and social dysfunction. Nature 477, 171-178. doi: 10.1038/nature 10360

Yui, K., Kawasaki, Y., Yamada, H., and Ogawa, S. (2016). Oxidative stress and nitric oxide in autism spectrum disorder and other neuropsychiatric disorders. CNS Neurol. Disord. Drug Targets 15, 587-596. doi: 10.2174/ 1871527315666160413121751

Conflict of Interest Statement: The authors declare that the research was conducted in the absence of any commercial or financial relationships that could be construed as a potential conflict of interest.

Copyright (c) 2018 Wang, Hausknecht, Shen and Haj-Dahmane. This is an openaccess article distributed under the terms of the Creative Commons Attribution License (CC BY). The use, distribution or reproduction in other forums is permitted, provided the original author(s) and the copyright owner(s) are credited and that the original publication in this journal is cited, in accordance with accepted academic practice. No use, distribution or reproduction is permitted which does not comply with these terms. 